\title{
The Side Effects of Not Being Vaccinated: Individual Risk and Vaccine Hesitancy Nationalism
}

\author{
David Shaw
}

Received: 28 July 2021 / Accepted: 16 September 2021 / Published online: 24 November 2021

(C) Journal of Bioethical Inquiry Pty Ltd. 2021

Keywords COVID-19 · Vaccination · Risk · Vaccine nationalism $\cdot$ Vaccine hesitancy

\section{Introduction: The Side Effects of Vaccines}

In this editorial I argue that the detection of extremely rare new side effects alone does not justify vaccine hesitancy among individuals or nations because any such stance must also consider the side effects of delaying vaccination. While there has been some limited discussion concerning the "risks" of not getting vaccinated, this language is insufficient; there are clear and identifiable effects of delaying vaccination on both the individual and the national level, many of them much more closely causatively linked to hesitancy than some of the supposed clotting "side effects" are to vaccines.

Despite some suggestions that vaccination against COVID-19 should be mandatory, governments globally have left the decision to individual citizens. In the United Kingdom, uptake has been very high, with over 90 per cent of priority groups receiving at

D. Shaw $(\bowtie)$

Institute for Biomedical Ethics, University of Basel, Basel,

Switzerland

e-mail: david.shaw@unibas.ch

D. Shaw

Care and Public Health Research Institute, Maastricht

University, Maastricht, The Netherlands least one vaccination by mid-April 2021. Yet some people prefer to either not get vaccinated at all or to "wait and see" how others fare, in case there are any unforeseen side effects. In light of widespread media coverage of blood clot concerns about two of the vaccines, such hesitancy might seem entirely justified. A person who initially refused vaccination might now say something like "my position has been vindicated; new side effects have been identified."

Vaccines do have side effects; the most common ones associated with COVID vaccines is a sore arm or fever in the forty-eight hours after vaccination. But those are not the side effects that people appear to be most worried about. Rather, the concerns related to rare yet deadly side effects that could not be detected in a clinical trial due to the relatively low number of participants.

In the case of the Astra Zeneca vaccine, concerns were initially raised about a very small number of blood clots among millions of vaccinations. These were rightly investigated; it transpired that most of them would have occurred in the population anyway and that the relationship was correlatory rather than causal, meaning it was not really a side effect. Indeed, any causal link seemed likely to reduce the risk of blood clots; the European Medical Agency stated that that the incidence of clots was actually lower in the vaccinated population than the general population (Mahase 2021a). However, there were even rarer cases of a combination of thrombosis and thrombocytopenia and bleeding and of disseminated 
intravascular coagulation (Mahase 2021a). These did seem to be side effects of the vaccine, though doubts remain as to its causative mechanism. (The Johnson \& Johnson vaccine seems to have a similar very rare side effect [EMA 2021]). So, although most of the reported blood clots were not actually side effects, but would have occurred anyway, there is a tiny increased risk of getting one of these unusual blood clots. But as we shall see, that rare side effect pales into insignificance next to the very real side effects of not getting vaccinated or delaying vaccination.

\section{The Side Effects of Individual Vaccine Hesitancy}

Unsurprisingly, the main effect (not really a side effect) of not being vaccinated is not being protected against COVID-19. This in turn has two main side effects; it increases your risk of getting COVID-19 and giving COVID-19 to someone else. And COVID19 , of course, increases one's risk of getting sick and dying. All these side effects endure as long as you are not vaccinated.

Often (indeed predominantly) the narrative about hesitancy is framed in terms of the pros and cons for oneself; "I' $m$ not at high risk of dying of COVID and I'm worried about the potential side effects of the vaccine, so I'll wait and see." This might seem reckless but nonetheless remains a matter of individual choice. But this stance is not only ethically problematic in terms of letting others carry the risk burden if there do turn out to be any side effects; it is also problematic in terms of posing risk to others; if I remain unvaccinated for several months there is a risk I'll get COVID, and if I do get it, there is a 50 per cent chance that I'll be asymptomatic and pass it on to someone else. And the "I' $m$ not at high risk so I'll wait" argument is even more problematic if the person advancing it is, for example, a health worker who risks giving the virus to highly vulnerable patients, potentially leading to their deaths. It is an open question whether health workers who refuse vaccination overlook this rather important side effect, or regard it as unpersuasive.

The side effects of getting COVID and passing it on among unvaccinated people are vastly more likely to occur than any blood clot side effect among the vaccinated. The risk of getting a blood clot is literally one in a million, and if you get COVID your risk of dying is about 1 in 100 (varying with age); also, even if you don't die, getting COVID increases your risk of blood clots. To take another example (and depending on prevalence), for those aged 70 and over, the risk of getting infected and then dying from COVID is approximately 1 in 1000 (Spieglelhalter 2020), as opposed to 1 in a million for a vaccine-related blood clot (Mahase 2021b). This ratio does change for younger people; for those aged 30, it's around 1 in 100,000 . But that still makes dying from COVID ten times more likely than getting a dangerous blood clot that is nonetheless treatable; furthermore, given the EMA's statement mentioned above, it is possible that those who delayed getting a vaccine were increasing rather than decreasing their risk of getting a blood clot. Nonetheless, the U.K. Joint Committee on Vaccination and Immunity (JCVI) in the United Kingdom ultimately decided that people aged under thirty should not be given the Astra Zeneca vaccine, and that those aged 30-39 should be able to choose an alternative, where one was available. This decision itself may have been motivated by a wish to avoid vaccine hesitancy rather than purely on the relative risks just discussed.

Furthermore, there are additional potential side effects related to vaccine hesitancy. As well as increasing the risk of infection for themselves and others, people who decide at the last minute not to get vaccinated increase wastage of vaccine doses which most often must be used on the day; thus they can deny or delay a vaccine dose to others who could have had it sooner. Each case of delayed vaccination also increases (marginally) the risk of another wave of COVID and of the risk that a new variant will arise in the population.

Finally, the risks associated with getting and passing on COVID are not the only side effects of not getting vaccinated. One notable side effect of vaccine hesitancy is that, if one's stance is communicated, it can create doubt and hesitancy in others, increasing the risk that other people will delay or refuse vaccination. (It is an unfortunate irony that hesitancy, like fake news, is highly communicable, just like a virus [Shaw 2020].)

Another point worth mentioning is that some people are hesitant about a particular vaccine but happy to receive another; for example, some people would prefer to get the Pfizer vaccine rather than the Astra Zeneca one, not because of blood clot concerns but 
because of perceived higher efficacy or even a perverse "vaccine nationalism" (Mercer 2021). Quite apart from the fact that allowing everyone to choose between vaccines would present substantial logistical challenges, there is a more serious problem here; anyone who wants to wait for a different vaccine exposes themselves to all the side effects mentioned above, out of concern not about vaccine side effects in general but about one vaccine in particular. In that sense, vaccine particularism makes even less sense in terms of risk analysis than "mainstream" vaccine hesitancy.

\section{The Side Effects of Vaccine Hesitancy Nationalism}

Just as the individual who is hesitant about vaccination cites concerns about side effects, so many nations-or rather, their medical agencies-paused vaccination with Astra Zeneca and Johnson \& Johnson vaccines "as a precaution" while clotting concerns were investigated. Note that the suggestion is not that such investigations should not take place; they can and must do so. However, pausing vaccination has such severe side effects on the public health response to the pandemic that it cannot be justified by concerns about a potential rare side effect that (at the time) could have been explained by correlation rather than causation-in which case it was not actually a side effect at all, as mentioned above.

The specific side effects of vaccine hesitancy nationalism are very similar to those that occur at the individual level, except exponentially worse because of the number of people directly affected. If vaccination pauses for one week, tens of thousands of people will remain unvaccinated during that period, meaning thousands more cases of COVID and hundreds more deaths; each of those cases is likely to lead to one or two others, depending on the local $\mathrm{R}$ number. In the European Union alone, eighteen countries including France, Germany, Italy, and Spain temporarily suspended vaccination with this vaccine (Holmes 2021), meaning that hundreds of thousands of people had their doses delayed and millions had their confidence in the vaccine unnecessarily shaken.

However, there is one highly significant side effect that occurs at the national level but not at the individual one: pausing a vaccination campaign also denies a dose to those who want and need it. If one person in a country refuses to get vaccinated and dies as a result, that is his problem; hopefully he did not take anyone with him. But if a country pauses vaccination, infection rates will rise, and many of those who get sick and die would have been perfectly happy to receive their vaccination. Vaccine hesitancy denies vaccines only to those who choose to delay vaccination; vaccine hesitancy nationalism denies vaccines to tens of thousands who want to be vaccinated. This failure to respect citizen's autonomy can also cause them great harm.

\section{Conclusion}

The irony of many concerns about vaccines is that they relate to "side effects" that are not really side effects but merely correlated events, while very serious causative side effects of refusing or delaying vaccination are not considered at all. Anyone who decides not to get vaccinated or to delay vaccination without considering the serious side effects of doing so makes a decision without sufficient information. Anyone who does delay vaccination despite considering that information would have to have a very good justification for doing so given the risk that it poses to their own and to public health. Similarly, any nation that pauses vaccination programmes while extremely rare side effects are investigated has probably caused hundreds if not thousands of avoidable deaths by increasing both the number of cases and the number of people who become hesitant about vaccines as a result. Anyone who is hesitant about getting vaccinated should consider that inaction also has side effects that can harm not only oneself, but also others.

\section{References}

European Medical Agency. 2021. COVID-19 Vaccine Janssen: EMA finds possible link to very rare cases of unusual blood clots with low blood platelets. https://www.ema. europa.eu/en/news/covid-19-vaccine-janssen-ema-findspossible-link-very-rare-cases-unusual-blood-clots-lowblood. Accessed May 26, 2021.

Holmes, O. 202.1Which European states have paused AstraZeneca jabs due to clotting concerns? The Guardian, March 16. https://www.theguardian.com/society/2021/ mar/15/which-european-states-have-paused-astrazenecajabs-due-to-clotting-concerns. Accessed May 26, 2021. 
Mahase, E. 2021a. COVID-19: AstraZeneca vaccine is not linked to increased risk of blood clots, finds European Medicine Agency. British Medical Journal 372: n774.

Mahase, E. 2021b. AstraZeneca vaccine: Blood clots are "extremely rare" and benefits outweigh risks, regulators conclude. British Medical Journal 373: n931.

Mercer, D. 2021. COVID-19: "I'll wait for the English vaccine"-How "nationalism" is affecting the fight against coronavirus. Sky News, February 6. https://news.sky.com/ story/covid-19-ill-wait-for-the-english-vaccine-how-natio nalism-is-affecting-the-fight-against-coronavirus- 12207 629. Accessed May 262021.
Shaw, D.M. 2020. Invisible enemies: Coronavirus and other hidden threats. Bioethical Inquiry 17: 531-534.

Spiegelhalter, D. 2020. Use of "normal" risk to improve understanding of dangers of COVID-19. British Medical Journal 370: m3259.

Publisher's Note Springer Nature remains neutral with regard to jurisdictional claims in published maps and institutional affiliations. 